Original Research Paper

\title{
A Polysaccharide-Rich Fraction Derived from Oviductus ranae with Antioxidant and Immunomodulatory Potentials
}

\author{
Xiaojin Yu, Xinyu Wang, Xinyu Liu, Lei Liu, Susu Zhang, Bowen Xiao, Lixue Zheng and Yang Zhang* \\ School of Biology and Food Engineering, Changshu Institute of Technology, Changshu 215500, China
}

\author{
Article history \\ Received: 12-08-2020 \\ Revised: 03-09-2020 \\ Accepted: 12-09-2020 \\ Corresponding Author: \\ Yang Zhang \\ School of Biology and Food \\ Engineering, Changshu \\ Institute of Technology, \\ Changshu 215500, China \\ Email: zhangyang@cslg.edu.cn
}

\begin{abstract}
Oviductus ranae, an animal-based traditional Chinese medicinal material listed in Chinese Pharmacopeia is widely consumed as tonic, approximately twenty Oviductus ranae-related functional foods have been approved on the market by National Medical Products Administration in China. Proteins are well-defined bioconstituents in Oviductus ranae, little information is available on its polysaccharides. In present work, a Polysaccharide-Rich fraction (POR-3) was fractionated from Oviductus ranae based on antioxidant activity-guided isolation and its structure was partially characterized as well as its immunomodulatory potential was explored. POR-3 belonged to a heteropolysaccharide-protein fraction mainly consisted of Man, GlcN, GalA, Gal and Fuc at a molar ratio of 1: 2.28: 2.40: 3.00: 2.23 with binding proteins content of $6.42 \pm 0.16 \%$. POR-3 possessed polydisperse property with MW of $999.586 \mathrm{kDa}$ and PDI of 4.09. The UV and FT-IR analysis demonstrated that structures of POR-3 correspond with the characteristics of polysaccharide-protein complex. POR-3 elicited higher scavenging capacities against hydroxyl and DPPH radicals and exerted potent in vitro immunomodulatory activity via promoting macrophages phagocytosis and via stimulating NO production as well as appeared no proliferation on macrophages. POR-3 possesses high potentials to be consumed as a medicine or functional food with antioxidant and immune modulator properties.
\end{abstract}

Keywords: Oviductus ranae, Polysaccharide-Rich Fraction, Antioxidant Activity-Guided Isolation, Immunomodulatory Activity

\section{Introduction}

Reactive Oxygen Species (ROS) play a dual role on immune system. Low ROS production induced by the activation of phagocyte NADPH oxidase 2 complex is considered to regulate immune response and cell proliferation and to control $\mathrm{T}$-cell autoreactivity (Lam et al., 2010). Nevertheless, excessive accumulation of ROS caused by harmful environmental stresses such as overconsumption of smoking and alcohol, long-term exposure to organic pollutants and heavy metals as well as X-ray irradiation could induce oxidative stress, thereby impairing the activated $\mathrm{T}$ cell and destroying the membrane structure of immune cell to cause immune disorder (Amir Aslani and Ghobadi, 2016; Hildeman et al., 2003; Srivastava et al., 2017).

Although endogenous antioxidants, including glutathione, superoxide dismutase, glutathione peroxidase and catalases usually exert certain scavenging capacities against ROS to decrease the damaging effects of oxidative stress (Ighodaro and Akinloye, 2018). However, levels of these endogenous antioxidants are always lower than the minimum concentrations demanded for scavenging ROS (Szuroczki et al., 2016). Therefore, it is of importance to supplement antioxidants exogenously, owing to their beneficial effects on oxidative stress-related disorders, particularly for immune dysfunction.

Natural polysaccharides belong to bio-macromolecules and their antioxidant potentials have been widely recognized (Huang et al., 2017; Wang et al., 2013). Recent studies have revealed that some of antioxidant polysaccharides usually elicit pronounced immunomodulatory properties, such as the polysaccharides from Gomphidius rutilus (Gao et al., 2013), Chenopodium quinoa (Hu et al., 2017), Ganoderma lucidum (Chen et al., 2009) and Punica granatum (Joseph et al., 2012). It is therefore feasible and 
meaningful to explore the immunomodulatory potentials of the polysaccharides with higher antioxidant capacities.

Rana dybowskii, one of the Chinese brown frogs is a famous economic animal widely farmed in Northeastern China, whose economic value mainly depends on its dried oviduct that is also called Oviductus ranae (OR), a traditional Chinese medicinal material listed in Chinese Pharmacopeia. Traditionally, OR was prescribed for the remedies of several ailments, including neurasthenia, insomnia, debilitation, climacteric syndrome and night sweat. OR differs from other traditional Chinese medicinal materials included in Chinese Pharmacopoeia, it is not considered as therapeutic agent but is usually consumed as active constituents for functional foods and nutraceuticals. To this day, approximately twenty ORrelated functional foods have been approved on the market by National Medical Products Administration (Zhang et al., 2019). OR comprises several bioactive components, including proteins, steroids, fatty acids and polysaccharides. Recent studies have found that OR shows immune enhancement, anti-fatigue, antiosteoporosis, anti-oxidation, anti-apoptosis, reproductive protection and estrogen-like effects. The proteins and steroids of OR have been well studied (Wang et al., 2010; Zhang et al., 2018). However, little information is available on the Polysaccharides from OR (POR).

In present work, an antioxidant polysaccharide fraction from OR was isolated from the purified POR based on antioxidant activity-guided fractionation, then the partial characterization as well as immunomodulatory potential were further explored. To the best of our knowledge, it is the first time to investigate POR, which is more likely to enrich the application of natural polysaccharides and to provide evidence and reference for the development and comprehensive utilization of OR as novel drugs and functional foods.

\section{Materials and Methods}

\section{Materials}

Freeze-dried OR was purchased from Jilin Huangzhihua Pharmaceutical Co., Ltd (Changchun, Jilin, China). It was authenticated by Prof. Dr. Limei Wang, School of Biology and Food Engineering, Changshu Institute of Technology (Changshu, Jiangsu, China). A specimen with voucher No. KLFB-2019-0016 was deposited at Suzhou Key Laboratory of Food Biotechnology, Changshu Institute of Technology (Changshu, Jiangsu, China).

Major reagents used for the in vitro antioxidant evaluation, including 1, 1-Diphenyl-2-Picrylhydrazyl $(\mathrm{DPPH})$, phenanthroline, ferrous sulfate $\left(\mathrm{FeSO}_{4}\right)$ and Vitamin C (VC) were obtained from Macklin Biochemical Co., Ltd. (Shanghai, China).
Standard sugars applied for monosaccharide composition analysis were from Sino-pharm Chemical Reagent Co., Ltd. (Shanghai, China), including $L$ Rhamnose (Rha), L-Fucose (Fuc), $D$-Glucose (GLu), $D$ fructose (Fru), $D$-Galactose (Gal), $D$-Mannose (Man), $D$ Arabinose (Ara), D-Galacturonic acid (GalA), DGlucosamine $(\mathrm{GlcN})$ and $D$-Xylose $(\mathrm{Xyl})$.

$\mathrm{T}$-series dextran standards used for molecular weight determination, including T-500, T-100, T-70, T-40 and $\mathrm{T}-10$ were provided by National Institutes for Food and Drug Control (Beijing, China).

Major reagents applied for immunomodulatory activity were purchased from Sigma Aldrich (St. Louis, MO, USA), including Fetal Bovine Serum (FBS), Lipopolysaccharide (LPS, from Escherichia coli O111:B4), 3-(4, 5dimethylthiazol-2-yl)-2, 5-diphenyltetrazolium bromide (MTT), Dimethylsulfoxide (DMSO), Phosphate Buffered Saline (PBS), Roswell Park Memorial Institute (RPMI)-1640 medium, neutral red, penicillin and streptomycin. Assay kit used for the determination of Nitric Oxide (NO) was from Nanjing Jiancheng Bioengineering Institute (Nanjing, Jiangsu, China).

Other materials, such as 1-Phenyl-3-Methyl-5Pyrazolone (PMP), Trifluoroacetic Acid (TFA), diethylaminoethyl (DEAE)-52 cellulose, papain, ethanol and sodium chloride $(\mathrm{NaCl})$ were obtained from Aladdin Bio-Chem Technology Co., Ltd. (Shanghai, China).

\section{Cell Culture}

Mouse leukaemic monocyte macrophage cell line RAW 264.7 (ATCC TIB-71) was cultured in RPMI 1640 medium containing $10 \% \mathrm{FBS}, 100 \mu \mathrm{g} / \mathrm{mL}$ streptomycin and $100 \mathrm{U} / \mathrm{mL}$ penicillin at $37^{\circ} \mathrm{C}$ in a humidified incubator with $5 \% \mathrm{CO}_{2}$.

\section{Extraction and Purification of Polysaccharides}

Freeze-dried OR was grinded into powders and sieved to 60 mesh, which were then soaked in petroleum ether to degrease at room temperature for $12 \mathrm{~h}$. According to the work reported by (Qin et al., 2018) with some modifications, ten grams of degreased OR powders were soaked in distilled water at liquid-to-solid ratio of 100: 1 for $12 \mathrm{~h}$, homogenated, adjusted to $\mathrm{pH} 6.8$ and incubated with papain in a ratio of $0.2 \%(\mathrm{w} / \mathrm{w})$ at $37^{\circ} \mathrm{C}$ in shaking water bath at 50 cycles/min for $2 \mathrm{~h}$. After extraction, the mixture was placed into boiling water for $10 \mathrm{~min}$ to inactivate papain, centrifugated at $10000 \mathrm{rpm}$ for $20 \mathrm{~min}$ to obtain the supernatant, which was neutralized with $1 \mathrm{M} \mathrm{NaOH}$, concentrated to a quarter of its volume and mixed with 3 -fold $95 \%(\mathrm{v} / \mathrm{v})$ ethanol at $4{ }^{\circ} \mathrm{C}$ for $12 \mathrm{~h}$. The precipitant was collected by centrifugation at $4000 \mathrm{rpm}$ for $10 \mathrm{~min}$ and freeze-dried to obtain crude polysaccharides, which were then subjected to a deproteinization by the Sevag method (Huang et al., 2010) for three times. After removal of Sevag reagent, the 
solution was dialyzed for three times, which was freezedried to yield purified polysaccharides. Polysaccharide content was determined by the phenol-sulfuric acid method with glucose as standard (Dubois et al., 1956). The standard curve was as follows:

$$
A=7.646 C+0.4064\left(R^{2}=0.9995\right)
$$

Where:

$A=$ Absorbance

$C=$ Polysaccharide content $(\mathrm{mg} / \mathrm{mL})$ linear range was from 0.02 to $0.12 \mathrm{mg} / \mathrm{mL}$

\section{Fractionation of Polysaccharides}

Purified polysaccharides were dissolved in distilled water and loaded onto a DEAE-52 cellulose column $(2.6 \times 60 \mathrm{~cm})$, followed by a successive elution with distilled water and $0.1 \sim 0.5 \mathrm{~mol} / \mathrm{L} \mathrm{NaCl}$ solution at a flow rate of $1 \mathrm{~mL} / \mathrm{min}$, respectively. The obtained eluates $(5 \mathrm{~mL} /$ tube) were collected and monitored by the phenol-sulfuric acid method. The elution fractions with relatively higher amounts were collected, dialyzed and lyophilized (Meng et al., 2018b).

\section{Chemical Analysis of POR-2 and POR-3}

The total carbohydrates, uronic acids and proteins of POR-2 and POR-3 were respectively measured by the phenol-sulfuric acid method (Dubois et al., 1956), by the $m$-hydroxy biphenyl method (Blumenkrantz and Gustav, 1973) and by the coomassie bright blue method (Bradford, 1976).

\section{Comparison of the In Vitro Antioxidant Activities}

The two fractions with relatively higher amounts (POR-2 and POR-3) were screened by comparison of the in vitro antioxidant activities to obtain the fraction with highest antioxidant activity.

\section{Hydroxyl Radical-Scavenging Activity}

POR-2 and POR-3 were respectively dissolved in distilled water to prepare solutions at different concentrations $(0.2 \sim 1.0 \mathrm{mg} / \mathrm{mL})$. Two milliliters of sample solution and $1 \mathrm{~mL}$ of PBS solution ( $\mathrm{pH} 7.4)$ containing $0.75 \mathrm{mmol} / \mathrm{L}$ phenanthroline were mixed, then $1 \mathrm{~mL}$ of $\mathrm{FeSO}_{4}$ solution $(0.75 \mathrm{mmol} / \mathrm{L})$ and and 1 $\mathrm{mL}$ of $\mathrm{H}_{2} \mathrm{O}_{2}$ solution $(0.12 \%$, v/v) were added. After incubation at $37^{\circ} \mathrm{C}$ for $60 \mathrm{~min}$, the absorbance at 536 $\mathrm{nm}\left(\mathrm{A}_{\mathrm{s}}\right)$ was determined. The mixtures without $\mathrm{H}_{2} \mathrm{O}_{2}$ and tested sample were served as normal control (Ac) and blank control $\left(\mathrm{A}_{0}\right)$, respectively. $\mathrm{VC}$ was used as positive control. The hydroxyl radical-scavenging rate was calculated according to the following equation (Zhang et al., 2018):
Hydroxyl radical - scavenging rate $(\%)$

$=\left(A_{s}-A_{0}\right) \times 100 /\left(A_{c}-A_{0}\right)$

\section{DPPH Radical-Scavenging Activity}

POR-2 and POR-3 were respectively dissolved in distilled water to prepare solutions at different concentrations $(0.2 \sim 1.0 \mathrm{mg} / \mathrm{mL})$. Two milliliters of sample solution and $2 \mathrm{~mL}$ of ethanol solution containing $0.1 \mathrm{mmol} / \mathrm{L} \mathrm{DPPH}$ were mixed, followed by a reaction in the dark for $30 \mathrm{~min}$ at room temperature, the absorbance at $517 \mathrm{~nm}\left(\mathrm{~A}_{\mathrm{s}}\right)$ was then measured. The mixtures in absence of DPPH and tested sample were used as normal control $\left(\mathrm{A}_{\mathrm{c}}\right)$ and blank control $\left(\mathrm{A}_{0}\right)$, respectively. VC was served as positive control. The DPPH radical-scavenging rate was obtained based on the following equation (Zhang et al., 2018):

$D P P H$ radical - scavenging rate $(\%)$

$=\left(A_{s}-A_{c}\right) \times 100 / A_{0}$

\section{Partial Characterization of POR-3}

\section{Molecular Weight}

Twenty microliters of $2 \mathrm{mg} / \mathrm{mL}$ POR-3 solution was filtered by using a $0.45 \mu \mathrm{m}$-pore Millipore filter, then the filtrate was injected into a Shodex SUGAR KS-804 column $(8.0 \times 300 \mathrm{~mm})$ and analyzed by a HighPerformance Liquid Chromatography (HPLC) (Elite P230IIHPLC, Elite Analytical Instruments Co. Ltd., Dalian, China) equipped with a Refractive Index Detector (RID) (RI2000, A, Schambeck SFD GmbH, Germany). Data was recorded and processed by using a N2000 GPC chromatographic work station. A series of dextran standards with different molecular weights were used to establish calibration curve. The analytical conditions were: Ultrapure water was served as mobile phase at a flow rate of $1.0 \mathrm{~mL} / \mathrm{min}$; the temperatures of column and RID were set as 50 and $35^{\circ} \mathrm{C}$, respectively and the run rate was set as $30 \mathrm{~min}$ (Hu et al., 2019).

\section{Monosaccharide Compositions}

Two milligrams of POR-3 was hydrolyzed by $2 \mathrm{~mL}$ of $2 \mathrm{M} \mathrm{TFA}$ at $110^{\circ} \mathrm{C}$ for $5 \mathrm{~h}$ to obtain monosaccharides. After removal of the residual TFA, the hydrolysate was reacted with $200 \mu \mathrm{L}$ of $0.5 \mathrm{M}$ PMP (dissolved in methanol) and $200 \mu \mathrm{L}$ of $0.3 \mathrm{M} \mathrm{NaOH}$ at $70^{\circ} \mathrm{C}$ for $1 \mathrm{~h}$. The reaction mixture was neutralized by $0.3 \mathrm{M} \mathrm{HCl}$ and extracted with chloroform for three times to obtain the solution containing PMP-labeled monosaccharides, which was then passed through a $0.45 \mu \mathrm{m}$-pore Millipore filter and injected into the Supersil ODS2 column $(5 \mu \mathrm{m}, 4.6 \times 250 \mathrm{~mm})$ of an Ultimate 3000 HPLC system (Thermo Fisher Scientific, Waltham, MA, USA) 
equipped with a diode array detector (DAD). The analytical conditions were: the mobile phase consisted of PBS (pH 6.8) and acetonitrile (82:18, v/v) at a flow rate of $0.8 \mathrm{~mL} / \mathrm{min}$; column temperature $-30^{\circ} \mathrm{C}$; detector wavelength-245 $\mathrm{nm}$; run rate-85 min. Different commercial monosaccharides were served as standards (Hu et al., 2019).

\section{Ultra Violet (UV) and Fourier Transform-Infrared (FT-IR) Analysis}

UV spectrum of POR-3 was determined by using a 2700 UV-vis spectrophotometer (Shimadzu, Kyoto, Japan) at $200 \sim 400 \mathrm{~nm}$. POR-3 was ground with dried $\mathrm{KBr}$ and pressed into pellets, then FT-IR spectrum of POR-3 was recorded on a FTIR-650 Fourier transforminfrared spectrophotometer (Gangdong Sci. and Tech. Development Co., Ltd, Tianjin, China) at $4000 \sim 400$ $\mathrm{cm}^{-1}$ (Wei et al., 2019).

\section{The In Vitro Immunomodulatory Activity of POR-3}

\section{Macrophages Proliferation Assay}

Effects of POR-3 on the proliferation of RAW264.7 cells were estimated by MTT method with some modifications ( $\mathrm{Hu}$ et al., 2019). Accordingly, RAW264.7 cells were seeded in 96-well plates containing RPMI-1640 medium and 10\% FBS. One hundred microliters of POR-3 solution ranging from 25 to $800 \mu \mathrm{g} / \mathrm{mL}$ were added and incubated at $37^{\circ} \mathrm{C}$ with $5 \% \mathrm{CO}_{2}$ for $24 \mathrm{~h}$. The complete medium without tested sample and the LPS at concentration of $25 \mu \mathrm{g} / \mathrm{mL}$ were served as negative and positive controls, respectively. After incubation, $10 \mu \mathrm{L}$ of MTT solution at concentration of $5 \mathrm{mg} / \mathrm{mL}$ were added to each well, followed by another incubation at $37^{\circ} \mathrm{C}$ with $5 \% \mathrm{CO}_{2}$ for $4 \mathrm{~h}$. Then supernatant was discarded and $100 \mu \mathrm{L}$ of DMSO were added to each well to dissolve formazan crystals. The absorbance at $490 \mathrm{~nm}$ was recorded by a microplate reader (Epoch2, BioTek, America).

\section{Macrophages Phagocytosis Assay}

Effects of POR-3 on the macrophages phagocytosis were evaluated by the neutral red uptake method with minor modifications ( $\mathrm{Hu}$ et al., 2019). In brief, RAW264.7 cells were seeded in 96-well plates and exposed to different concentrations of POR-3 solution or complete medium alone (negative control) or LPS solution (positive control) and incubated for $24 \mathrm{~h}$. After that, the supernatant was transferred into another 96well plate for the determination of NO content (see section of NO assay) and non-adherent cells were removed by washing twice with $\mathrm{PBS}$, followed by adding $100 \mu \mathrm{L}$ of neutral red solution to each well and by being incubated for $1 \mathrm{~h}$. After removal of the supernatant and excessive neutral red solution, cells were washed twice with PBS. Then, $100 \mu \mathrm{L}$ of cell lysate $(1.0 \mathrm{~mol} / \mathrm{L}$ acetic acid: Ethanol = 1: 1$)$ were added and kept overnight at room temperature and the absorbance at $490 \mathrm{~nm}$ was determined.

\section{NO assay}

Effects of POR-3 on NO release were appraised according to the method described in NO assay kit (Hu et al., 2019). In short, the supernatant was mixed with equivalent amounts of Griess reagent I (1\% sulfanilamide) and Griess reagent II $(0.1 \% \quad \mathrm{~N}-1$ naphthylethylenediamine dihydrochloride in $5 \%$ phosphoric acid) at room temperature for $10 \mathrm{~min}$. The absorbance at $540 \mathrm{~nm}$ was recorded and nitrite concentration was calculated by reference to sodium nitrite standards.

\section{Statistical Analysis}

Data was expressed as means \pm SD (standard deviation). Statistical analysis was conducted with t-test or one-way Analysis Of Variance (ANOVA) by using SPSS 19.0 software (SPSS Inc., Chicago, USA). $P<$ 0.05 was deemed as statistically significant.

\section{Results and Discussion}

\section{Extraction and Purification of Polysaccharides}

Extraction yield of crude POR was $5.09 \pm 0.21 \%$ and polysaccharide content was found to be $23.08 \pm 1.56 \%$. After deproteinization and dialysis, polysaccharide content in purified POR was upgraded to $55.42 \pm 1.21 \%$.

\section{Fractionation and Chemical Analysis of Polysaccharide Fractions}

Purified POR was fractionated by a DEAE-52 cellulose column chromatography and the elution curve was presented in Fig. 1. Three fractions, namely POR-1, POR-2 and POR-3 were fractionated with 0.1 to $0.3 \mathrm{M}$ $\mathrm{NaCl}$ solution. The two major fractions, accordingly POR-2 and POR-3 were collected, respectively. After dialysis and lyophilization, their chemical constituents were roughly determined, which were summarized in Table 1. It can be seen that contents of total carbohydrates $(P<0.05)$ and uronic acids $(P<0.01)$ in POR-3 were significantly higher than those in POR-2, while content of proteins in POR-3 was remarkably lower $(P<0.05)$ than that in POR-2. It has been reported that uronic acids enable polysaccharides negatively charged (Sun et al., 2018) and POR-3 contained more uronic acids than that of POR-2 (Table 1), which may be the reason that fractionation of POR-3 required relatively higher concentration of $\mathrm{NaCl}$ solution as eluent.

Values were expressed as means \pm SD for three experiments. Different superscript letters in the same column represented statistically significant differences $(P<0.05$, or $P<0.01)$. 
Table 1: General chemical constituents in the major polysaccharide fractions of purified POR

\begin{tabular}{llll}
\hline Fraction & Total carbohydrates $/ \%$ & Uronic acids/\% & Proteins/\% \\
\hline POR-2 & $75.05 \pm 1.24^{\mathrm{a}}$ & $4.91 \pm 0.28^{\mathrm{a}}$ & $8.39 \pm 0.29^{\mathrm{a}}$ \\
POR-3 & $80.87 \pm 1.17^{\mathrm{b}}$ & $10.19 \pm 0.43^{\mathrm{b}}$ & $6.42 \pm 0.16^{\mathrm{b}}$ \\
\hline
\end{tabular}

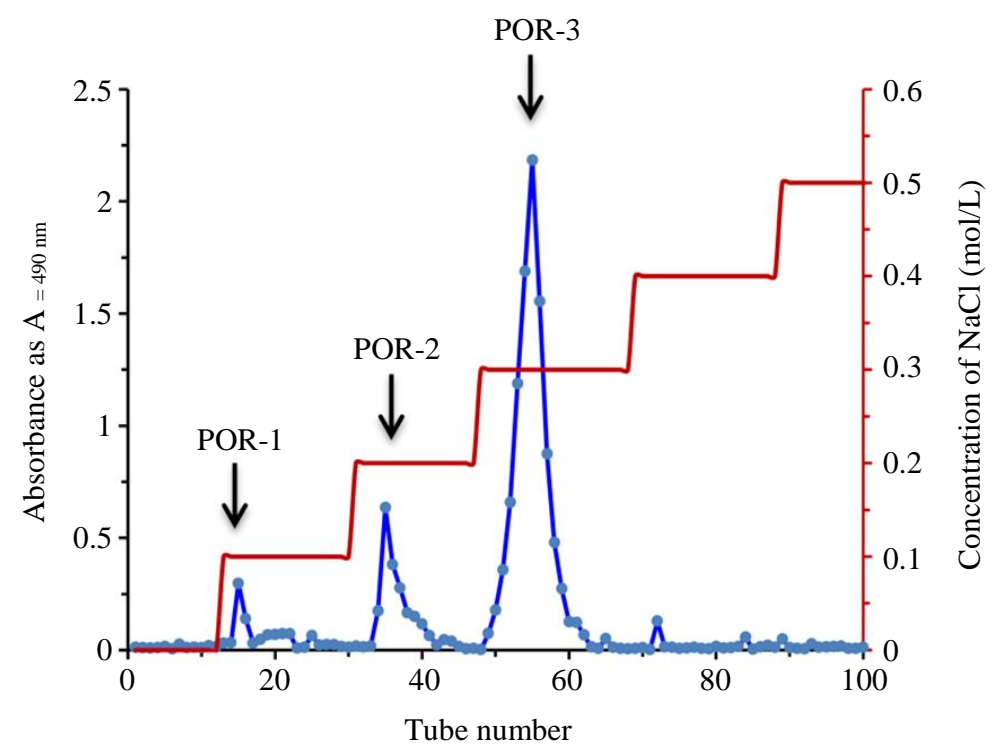

Fig. 1: The elution curve of purified POR on DEAE column chromatography

(A)

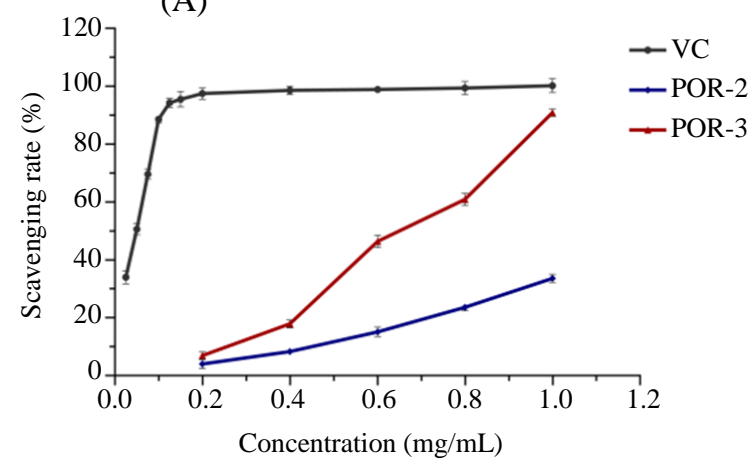

(B)

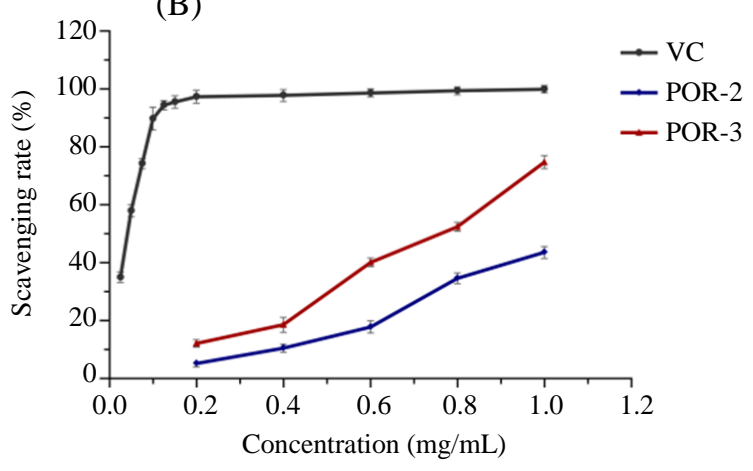

Fig. 2: The comparison of in vitro antioxidant capacities of POR-2 and POR-3 using VC as positive control. (A) Hydroxyl radicalscavenging capacity; (B) DPPH radical-scavenging capacity

\section{Comparison of the In Vitro Antioxidant Activities}

The in vitro scavenging capacities of POR-2 and POR-3 against hydroxyl and DPPH radicals were appraised and compared to define the polysaccharide fraction with relatively higher antioxidant activity. As shown in Fig. 2A, the hydroxyl radical-scavenging activities of POR-2 and POR-3 were elevated with the increase of sample concentration ranging from 0.2 $1.0 \mathrm{mg} / \mathrm{mL}$, the highest scavenging rates of POR-2 and POR-3 against hydroxyl radical were $33.65 \pm 1.39 \%$ and $90.94 \pm 1.25 \%$, respectively. The $\mathrm{IC}_{50}$ (half-inhibitory concentration) value of POR-3 against hydroxyl radical was $0.65 \pm 0.008 \mathrm{mg} / \mathrm{mL}$, significantly lower $(P<0.01)$ than that of POR-2 $(1.49 \pm 0.05 \mathrm{mg} / \mathrm{mL})$, but higher $(P<$ $0.01)$ than that of VC $(0.045 \pm 0.0008 \mathrm{mg} / \mathrm{mL})$.

As shown in Fig. $2 \mathrm{~B}$, in the range of $0.2 \sim 1.0$ $\mathrm{mg} / \mathrm{mL}$, POR-2 and POR-3 also elicited scavenging activities against DPPH radical, which were raised with the increase of sample concentration, the highest scavenging rates of POR-2 and POR-3 against DPPH radical reached $43.63 \pm 2.04 \%$ and $74.78 \pm 2.22 \%$, respectively. The $\mathrm{IC}_{50}$ value of POR-3 against DPPH radical was $0.73 \pm 0.01 \mathrm{mg} / \mathrm{mL}$, still markedly lower $(P<$ $0.01)$ than that of POR-2 $(1.15 \pm 0.03 \mathrm{mg} / \mathrm{mL})$, but higher $(P<0.01)$ than that of $\mathrm{VC}(0.037 \pm 0.004 \mathrm{mg} / \mathrm{mL})$. 
These results indicated that POR-3 outperforms POR2 on scavenging activities against hydroxyl and DPPH radicals, which might be contributed partially by the relatively higher content of uronic acids in POR-3 (Table 1), due to the fact that uronic acids are well acknowledged as an important role in antioxidant activity (He et al., 2016).

\section{Partial characterization of POR-3}

Considering that POR-3 exhibits a stronger in vitro antioxidant activity deserving of deep study, POR-3 was therefore selected for further investigations.

POR-3 was a heteropolysaccharide fraction that mainly consisted of Man, GlcN, GalA, Gal and Fuc at a molar ratio of $1: 2.28: 2.40: 3.00: 2.23$. The weightaverage molecular weight (MW) was $999.586 \mathrm{kDa}$ and number-average Molecular weight (MN) was $244.366 \mathrm{kDa}$. Polydispersity Index (PDI) calculated by $\mathrm{MW} / \mathrm{MN}$ was found to be 4.09 , indicating that POR-3 belongs to polydisperse polysaccharide fraction (Castro et al., 2016).

UV spectrum of POR-3 was presented in Fig. 3, from it, a noticeable absorption peak can be observed at around $280 \mathrm{~nm}$, suggesting the existence of residual free proteins or conjugated proteins in POR-3 (Meng et al., 2018a), which was consistent with the findings in Table 1, where both POR-2 and POR-3 were observed to contain certain amounts of proteins, further corroborating the suspicion of conjugated proteins, because free proteins can be basically removed by the Sevag reagent (Gu et al., 2020).

FT-IR spectrum of POR-3 was showed in Fig. 4, to be specific, broad peak at $3417 \mathrm{~cm}^{-1}$ was assigned to the stretching vibration of $-\mathrm{OH}$, the asymmetric and symmetric stretching vibrations of $-\mathrm{CH}_{2}$ - were registered at 2925 and $2854 \mathrm{~cm}^{-1}$, respectively. The strong absorption peaks at 1646 and $1548 \mathrm{~cm}^{-1}$, representing the stretching vibrations of carbonyl group, amides I and II, were from uronic acids and bound proteins in POR-3 (Liu et al., 2013; 2018), which were in accordance with the results reported by $(\mathrm{Gu}$ et al., 2020). Three absorption peaks in the range of $1200 \sim 1000 \mathrm{~cm}^{-1}$, namely 1121,1078 and $1036 \mathrm{~cm}^{-1}$ were assigned to the stretching modes of pyran ring, implying the presence of C-O-C glycosidic band (Liu et al., 2018). The weak absorption peak at about $765 \mathrm{~cm}^{-1}$ was recorded as the ring stretching and/or deformation of $\alpha-D-(1-4)$ and $\alpha-D-(1-6)$ bonds (Gu et al., 2020). These results showed that POR-3 possesses the characteristic groups of polysaccharides and could be classified as a kind of pyranose.

\section{The In Vitro Immunomodulatory Activity of POR-3}

\section{Effects of POR-3 on Macrophages Proliferation}

Macrophages play a crucial role in immune system, where they can activate innate immune and fight against infection and inflammation (Sun et al., 2015). As shown in Fig. 5, there were no significant differences $(P>0.05)$ in macrophages proliferation between NC and POR-3 treated groups even up to the concentration of $800 \mu \mathrm{g} / \mathrm{mL}$. The results showed that POR-3 has no proliferative or suppressive effects on macrophages, implying that treatment of POR-3 in the concentration range of $25 \sim 800 \mu \mathrm{g} / \mathrm{mL}$ could be no toxicity to macrophages.

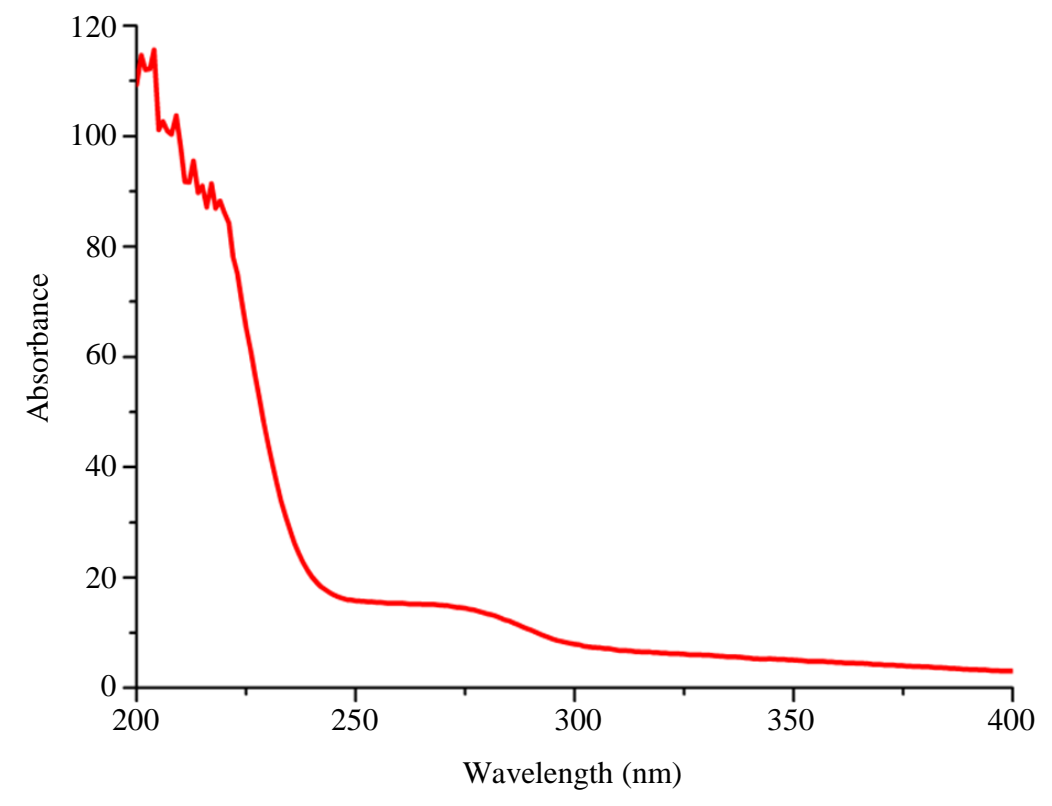

Fig. 3: UV spectrum of POR-3 


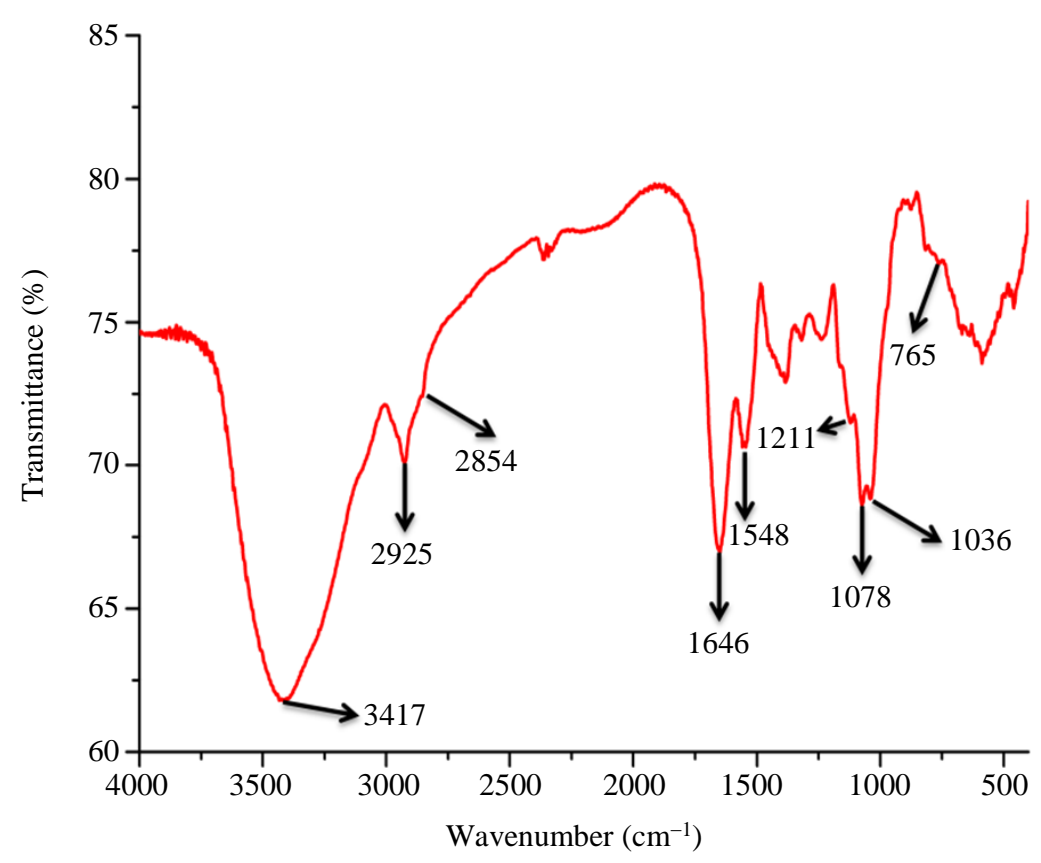

Fig. 4: FT-IR spectrum of POR-3

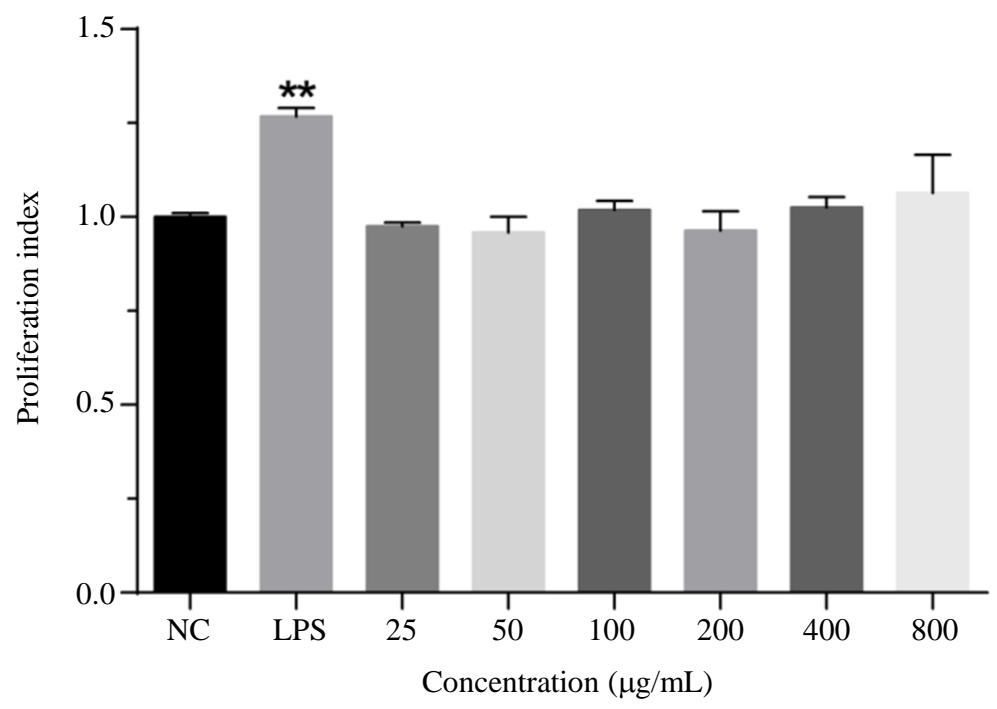

Fig. 5: Effects of POR-3 on macrophages proliferation. ${ }^{* *} P<0.01$ compared with the NC (negative control)

\section{Effects of POR-3 on Macrophages Phagocytosis}

Phagocytosis, a fundamental cellular process that has been defined as the leading function of macrophages is recognized as a major mechanism of immune responses to pathogens (Meng et al., 2018a). As shown in Fig. 6, when compared with NC, significant differences $(P<$ 0.01) in phagocytic index were found in all POR-3 treated groups, which was raised with the increase of concentration ranging from 25 to $50 \mu \mathrm{g} / \mathrm{mL}$. When the concentration of POR-3 was $50 \mu \mathrm{g} / \mathrm{mL}$, phagocytic index reached highest level of $1.32 \pm 0.074$, which was almost equivalent to that of LPS $(1.31 \pm 0.017)$, after that, it was not elevated with the increase of sample concentration, no remarkable differences $(P>0.05)$ were observed in the concentration range of $50 \sim 800 \mu \mathrm{g} / \mathrm{mL}$. These results indicated that POR-3 exerts potent promoting effects on macrophages phagocytosis, however, likely due to the inability of proliferating macrophages, this beneficial activity would be not enhanced in a dose-dependent manner. Exact reasons and mechanisms should be explored in the near future. 


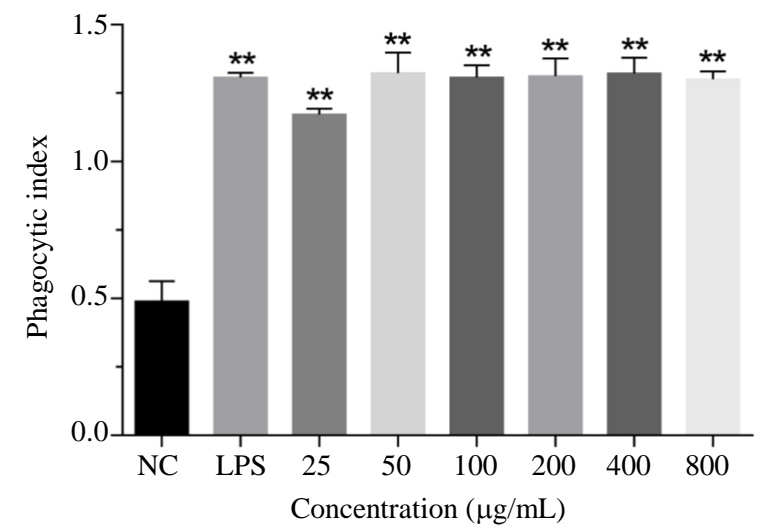

Fig. 6: Effects of POR-3 on macrophages phagocytosis. ${ }^{* *} P<$ 0.01 compared with the NC (negative control)

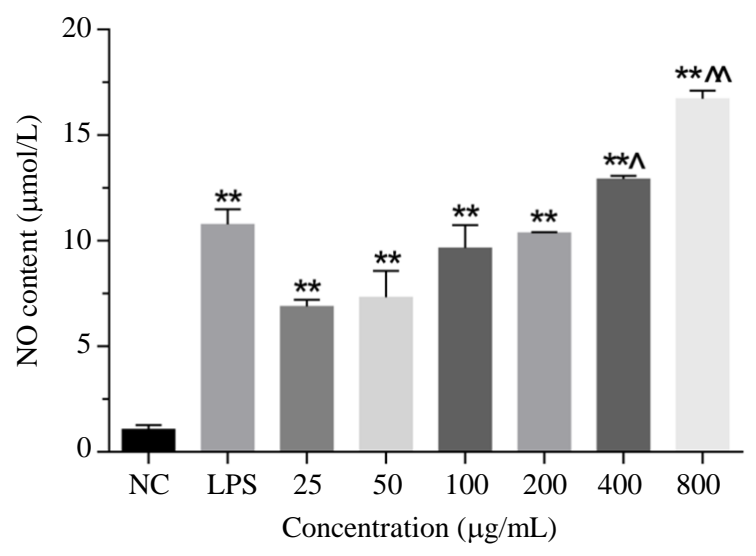

Fig. 7: Effects of POR-3 on NO release. ${ }^{* *} P<0.01$ compared with the NC (negative control). ${ }^{\wedge} P<0.05$ compared with LPS. ${ }^{\wedge \wedge} P<0.01$ compared with LPS

\section{Effects of POR -3 on $\mathrm{NO}$ release}

$\mathrm{NO}$ is one of the vital signaling molecules that widely participate in diverse physiological processes, particularly in defending against the invasion of pathogens. The release of NO usually depends on exogenous stimulating agents (Li et al., 2017). As shown in Fig. 7, POR-3 simulated NO production in a dosedependent manner $(P<0.01)$ in the concentration range of $25 \sim 800 \mu \mathrm{g} / \mathrm{mL}$. The NO content in RAW 264.7 cells being treated with POR-3 in the concentration of 800 $\mu \mathrm{g} / \mathrm{mL}$ reached the highest level of $16.72 \pm 0.38 \mu \mathrm{mol} / \mathrm{L}$, which was evidently higher $(P<0.01)$ than that of LPS $(10.77 \pm 0.72 \mu \mathrm{mol} / \mathrm{L})$. Significant difference $(P<0.05)$ in NO production was even observed between POR-3 in the concentration of $400 \mu \mathrm{g} / \mathrm{mL}$ and LPS $(12.93 \pm 0.14$ $\mu \mathrm{mol} / \mathrm{L}$ for POR-3 Vs. $10.77 \pm 0.72 \mu \mathrm{mol} / \mathrm{L}$ for LPS). The results demonstrated that POR-3 equally elicits potent stimulating effects on NO production, suggesting a potential of being used for the remedies of various disorders in addition to immune dysfunction, owing to the reason that $\mathrm{NO}$ needs to be involved in the regulation of a widely ranges of pathophysiological processes (Achike and Kwan, 2003).

In present investigation, POR-3 exhibited potent in vitro immunomodulatory activities via promoting macrophages phagocytosis and via stimulating NO production, as well as appeared no toxicity to macrophages.

Immunomodulatory activity is one of the most beneficial functions of polysaccharides (Cao et al., 2018). Althrough a broader range of structure-function relationships of immunostimulatory polysaccharides has not been well established owing to the insufficiency of precise separation and full characterization, actually several physicochemical properties such as monosaccharides composition, uronic acids content and molecular weights distribution have been recognized to be highly associated with the immunomodulatory activity of polysaccharides (Hu et al., 2019).

It has reported that polysaccharides with relatively abundant monosaccharides including Arabinose (Ara), Xylose (Xyl), Mannose (Man), Galactose (Gal), Glucose (Glu) and Fucose (Fuc) commonly exhibit higher macrophage stimulatory activities, because on the membrane of macrophages, specific receptors that can recognize these monosaccharides such as Complement Receptor 3 (CR3), Mannose Receptor (MR), Toll-Like Receptors (TLRs), Scavenger Receptors (SRs) and Mannose/Fucose Receptor (MFR) are highly expressed (Ferreira et al., 2015; Lo et al., 2007; Meng et al., 2018a; Shepherd et al., 1982). In present investigation, POR-3 mainly comprised Man, GlcN, GalA, Gal and Fuc at a molar ratio of 1: 2.28: 2.40: 3.00: 2.23 and total moles of the reported immunostimulatory monosaccharides were 6.23 , accounting for approximately $57 \%$.

As mentioned above, uronic acids can enable polysaccharides negatively charged (Gu et al., 2020), which could enhance the interactions with immune-related receptors and immunoglobulins (Jimenez-Dalmaroni et al., 2009; Manjula et al., 1982). As shown in Table 1, the content of uronic acids in POR-3 was up to $10.19 \pm 0.43 \%$, which was in accordance with the monosaccharides composition analysis, where GalA was observed to be present in POR-3.

With regard to the effects of molecular weight distribution on the immunomodulatory activity of polysaccharides, different voices can be heard. Im et al. (2005) found that polysaccharides with molecular weights ranging from 5 to $400 \mathrm{kDa}$ exhibit the most potent macrophage-activating activity using modified Aloe polysaccharides as model members. However, to summarize and analyze the molecular weight distributions of immunomodulatory polysaccharides reported by literatures, it can be inferred that the immunomodulatory activity of polysaccharides seems to be less connected with their molecular weights (Ferreira et al., 2015). This 
controversial issue might be explained by the different immune system activation modes of different immunomodulatory polysaccharides. As stated above, several receptors such as CR3, MR, TLRs, SRs and MFR are highly expressed on the membrane of macrophages and they can recognize the specific monosaccharides of immunomodulatory polysaccharides to trigger immune system (Ferreira et al., 2015; Shepherd et al., 1982). While, for certain non-specific immunomodulatory polysaccharides, entrance into macrophages would be the first step of action, which requires that polysaccharides should possess lower molecular weights, thereby facilitating the entrance via transporters such as glucose transporters and oligosaccharide transporters (Fu et al., 2004; Sun et al., 2013). In this study, the MW of POR-3 was found to be $999.586 \mathrm{kDa}$, larger than $400 \mathrm{kDa}$ (Im et al., 2005), combining its monosaccharides composition, it can be conjectured that POR-3 would elicit immunomodulatory activity via binding to certain specific receptors expressed on the surface of macrophages, particularly MR and MFR. Nevertheless, details remain to be unravelled and deepened.

In addition, due to the well-known immuneenhancing function of proteins (Mason et al., 2014), the binding proteins in POR-3 could also potentiate its immunomodulatory activity, which has been confirmed by Zhang et al. (2014) who observed that polysaccharide-protein complex from Lycium barbarum L. showed higher immunological enhancement than that of polysaccharide. Furthermore, the branched structure, spatial conformation and the branching degree equally affect the immunomodulatory activity of polysaccharides (Ferreira et al., 2015). It is therefore important to explore the structure-immunoregulatory function relationship of POR-3 in future investigations.

\section{Conclusion}

In presnt investigation, three fractions POR-1 $\sim 3$ were isolated from the purified polysaccharides of $\mathrm{OR}$, the two major fractions POR-2 and POR-3 were compared on the aspect of in vitro antioxidant activities to obtain the fraction deserving of further studies. POR-3 outperformed POR-2 on scavenging activities against hydroxyl and DPPH radicals, thus POR-3 was analyzed and partially characterized. Results indicated that POR-3 belongs to a polydisperse heteropolysaccharide-protein fraction. In RAW 264.7 macrophages, POR-3 elicited potent promoting effects on macrophages phagocytosis and NO release, but no proliferating or inhibiting effects on macrophages growth, exhibiting a desirable safety property for macrophages. Finally, the probable structure-immunomodulatory function relationships and underlying mechanisms of POR-3 were discussed and speculated. It is hoped that present work might provide evidence that animal-derived polysaccharides could be an emerging source of bioactive components for nutraceutical and medicinal purposes.

\section{Funding Information}

This work was supported by the project of Suzhou Municipal Science and Technology Bureau [grant number SNG201906] and the programs of Changshu Institute of Technology [grant numbers TD2025, XJDC2019218 and CSLGJG2018046].

\section{Author's Contributions}

All authors equally contributed in this work.

\section{Ethics}

This article is original and contains unpublished material. The corresponding author confirms that all of the other authors have read and approved the manuscript and no ethical issues involved.

\section{References}

Achike, F. I., \& Kwan, C. Y. (2003). Nitric oxide, human diseases and the herbal products that affect the nitric oxide signalling pathway. Clin Exp Pharmacol Physiol, 30(9), 605-615.

Amir Aslani, B., \& Ghobadi, S. (2016). Studies on oxidants and antioxidants with a brief glance at their relevance to the immune system. Life Sci, $146,163-173$.

Blumenkrantz, N., \& Gustav, A. H. (1973). New method for quantitative determination of uronic acids. Anal Biochem, 54(2), 484-489.

Bradford, M. M. (1976). A rapid and sensitive method for the quantitation of microgram quantities of protein utilizing the principle of protein-dye binding. Anal Biochem, 72, 248-254.

Cao, J., Tang, D., Wang, Y., Li, X., Hong, L., \& Sun, C. (2018). Characteristics and immune-enhancing activity of pectic polysaccharides from sweet cherry (Prunus avium). Food Chem, 254, 47-54.

Castro, N., Durrieu, V., Raynaud, C., \& Rouilly, A. (2016). Influence of DE-value on the physicochemical properties of maltodextrin for melt extrusion processes. Carbohydr Polym, 144, 464-473.

Chen, X. P., Chen, Y., Li, S. B., Chen, Y. G., Lan, J. Y., \& Liu, L. P. (2009). Free radical scavenging of Ganoderma lucidum polysaccharides and its effect on antioxidant enzymes and immunity activities in cervical carcinoma rats. Carbohydr Polym, 77(2), 389-393. 
Dubois, M., Gilles, K. A., Hamilton, J. K., Rebers, P. A., \& Smith, F. (1956). Colorimetric method for determination of sugars and related substances. Anal Chem, 28(3), 350-356.

Ferreira, S. S., Passos, C. P., Madureira, P., Vilanova, M., \& Coimbra, M. A. (2015). Structure-function relationships of immunostimulatory polysaccharides: A review. Carbohydr Polym, 132, 378-396.

Fu, Y., Maianu, L., Melbert, B. R., \& Garvey, W. T. (2004). Facilitative glucose transporter gene expression in human lymphocytes, monocytes and macrophages: a role for GLUT isoforms 1, 3 and 5 in the immune response and foam cell formation. Blood Cells Mol Dis, 32(1), 182-190.

Gao, C., Wang, Y., Wang, C., \& Wang, Z. (2013). Antioxidant and immunological activity in vitro of polysaccharides from Gomphidius rutilus mycelium. Carbohydr Polym, 92(2), 2187-2192.

Gu, J., Zhang, H., Zhang, J., Wen, C., Ma, H., Duan, Y., \& He, Y. (2020). Preparation, characterization and bioactivity of polysaccharide fractions from Sagittaria sagittifolia L. Carbohydr Polym, 229, 115355.

He, P., Zhang, A., Zhang, F., Linhardt, R. J., \& Sun, P. (2016). Structure and bioactivity of a polysaccharide containing uronic acid from Polyporus umbellatus sclerotia. Carbohydr Polym, 152, 222-230.

Hildeman, D. A., Mitchell, T., Kappler, J., \& Marrack, P. (2003). T cell apoptosis and reactive oxygen species. J Clin Invest, 111(5), 575-581.

Hu, Y., Zhang, J., Zou, L., Fu, C., Li, P., \& Zhao, G. (2017). Chemical characterization, antioxidant, immune-regulating and anticancer activities of a novel bioactive polysaccharide from Chenopodium quinoa seeds. Int J Biol Macromol, 99, 622-629.

Hu, Z., Zhou, H., Li, Y., Wu, M., Yu, M., \& Sun, X. (2019). Optimized purification process of polysaccharides from Carex meyeriana Kunth by macroporous resin, its characterization and immunomodulatory activity. Int J Biol Macromol, $132,76-86$

Huang, G., Mei, X., \& Hu, J. (2017). The antioxidant activities of natural polysaccharides. Curr Drug Targets, 18(11), 1296-1300.

Huang, G. L., Yang, Q., \& Wang, Z. B. (2010). Extraction and deproteinization of mannan oligosaccharides. Z Naturforsch C J Biosci, 65(5-6), 387-390.

Ighodaro, O. M., \& Akinloye, O. A. (2018). First line defence antioxidants-superoxide dismutase (SOD), catalase (CAT) and glutathione peroxidase (GPX): Their fundamental role in the entire antioxidant defence grid. Alexandria Journal of Medicine, 54(4), 287-293.
Im, S. A., Oh, S. T., Song, S., Kim, M. R., Kim, D. S., Woo, S. S., Lee, C. K. (2005). Identification of optimal molecular size of modified Aloe polysaccharides with maximum immunomodulatory activity. Int Immunopharmacol, 5(2), 271-279.

Jimenez-Dalmaroni, M. J., Xiao, N., Corper, A. L., Verdino, P., Ainge, G. D., Larsen, D. S., Wilson, I. A. (2009). Soluble CD36 ectodomain binds negatively charged diacylglycerol ligands and acts as a co-receptor for TLR2. PLoS One, 4(10), e7411.

Joseph, M. M., Aravind, S. R., Varghese, S., Mini, S., \& Sreelekha, T. T. (2012). Evaluation of antioxidant, antitumor and immunomodulatory properties of polysaccharide isolated from fruit rind of Punica granatum. Mol Med Rep, 5(2), 489-496.

Lam, G. Y., Huang, J., \& Brumell, J. H. (2010). The many roles of NOX2 NADPH oxidase-derived ROS in immunity. Semin Immunopathol, 32(4), 415-430.

Li, Q. M., Wang, J. F., Zha, X. Q., Pan, L. H., Zhang, H. L., \& Luo, J. P. (2017). Structural characterization and immunomodulatory activity of a new polysaccharide from jellyfish. Carbohydr Polym, 159, 188-194.

Liu, H. J., Xu, C. H., Zhou, Q., Wang, F., Li, W. M., Ha, Y. M., \& Sun, S. Q. (2013). Analysis and identification of irradiated Spirulina powder by a three-step infrared macro-fingerprint spectroscopy. Radiat Phys Chem, 85, 210-217.

Liu, Q., Ge, X., Chen, L., Cheng, D., Yun, Z., Xu, W., \& Shao, R. (2018). Purification and analysis of the composition and antioxidant activity of polysaccharides from Helicteres angustifolia L. Int J Biol Macromol, 107(Pt B), 2262-2268.

Lo, T. C., Jiang, Y. H., Chao, A. L., \& Chang, C. A. (2007). Use of statistical methods to find the polysaccharide structural characteristics and the relationships between monosaccharide composition ratio and macrophage stimulatory activity of regionally different strains of Lentinula edodes. Anal Chim Acta, 584(1), 50-56.

Manjula, B. N., Potter, M., \& Glaudemans, C. P. J. (1982). The interaction of mouse myeloma immunoglobulin S15 with negatively charged polysaccharide antigens. Mol Immunol, 19(7), 913-923.

Mason, A. P., Smilanich, A. M., \& Singer, M. S. (2014). Reduced consumption of protein-rich foods follows immune challenge in a polyphagous caterpillar. J Exp Biol, 217(Pt 13), 2250-2260.

Meng, F., Li, Q., Qi, Y., He, C., Wang, C., \& Zhang, Q. (2018a). Characterization and immunoregulatory activity of two polysaccharides from the root of Ilex asprella. Carbohydr Polym, 197, 9-16.

Meng, Q., Wang, Y., Chen, F., Xiao, T., \& Zhang, L. (2018b). Polysaccharides from Diaphragma juglandis fructus: Extraction optimization, antitumor and immune-enhancement effects. Int $\mathbf{J}$ Biol Macromol, 115, 835-845. 
Qin, Y., Yuan, Q., Zhang, Y., Li, J., Zhu, X., Zhao, L., Zhao, J. (2018). Enzyme-assisted extraction optimization, characterization and antioxidant activity of polysaccharides from sea cucumber Phyllophorus proteus. Molecules, 23(3), 590.

Shepherd, V. L., Campbell, E. J., Senior, R. M., \& Stahl, P. D. (1982). Characterization of the mannose/fucose receptor on human mononuclear phagocytes. J Reticuloendothel Soc, 32(6), 423-431.

Srivastava, S., Singh, D., Patel, S., \& Singh, M. R. (2017). Role of enzymatic free radical scavengers in management of oxidative stress in autoimmune disorders. Int J Biol Macromol, 101, 502-517.

Sun, D., Wang, Y., Tan, F., Fang, D., Hu, Y., Smith, D. E., \& Jiang, H. (2013). Functional and molecular expression of the proton-coupled oligopeptide transporters in spleen and macrophages from mouse and human. Mol Pharm, 10(4), 1409-1416.

Sun, H., Zhang, J., Chen, F., Chen, X., Zhou, Z., \& Wang, H. (2015). Activation of RAW264.7 macrophages by the polysaccharide from the roots of Actinidia eriantha and its molecular mechanisms. Carbohydr Polym, 121, 388-402.

Sun, Y., Hou, S., Song, S., Zhang, B., Ai, C., Chen, X., \& Liu, N. (2018). Impact of acidic, water and alkaline extraction on structural features, antioxidant activities of Laminaria japonica polysaccharides. Int J Biol Macromol, 112, 985-995.

Szuroczki, D., Koprivnikar, J., \& Baker, R. L. (2016). Dietary antioxidants enhance immunocompetence in larval amphibians. Comp Biochem Physiol A Mol Integr Physiol, 201, 182-188.
Wang, H., Liu, Y. M., Qi, Z. M., Wang, S. Y., Liu, S. X., Li, X., . . . Xia, X. C. (2013). An overview on natural polysaccharides with antioxidant properties. Curr Med Chem, 20(23), 2899-2913.

Wang, Y., Wang, L., Hu, Y., Zhang, L., \& Wang, Z. (2010). Isolation and identification of two steroid compounds from Oviductus ranae. Nat Prod Res, 24(16), 1518-1522.

Wei, E., Yang, R., Zhao, H., Wang, P., Zhao, S., Zhai, W., Zhou, H. (2019). Microwave-assisted extraction releases the antioxidant polysaccharides from seabuckthorn (Hippophae rhamnoides L.) berries. Int J Biol Macromol, 123, 280-290.

Zhang, X., Li, Y., Cheng, J., Liu, G., Qi, C., Zhou, W., \& Zhang, Y. (2014). Immune activities comparison of polysaccharide and polysaccharide-protein complex from Lycium barbarum L. Int J Biol Macromol, 65, 441-445.

Zhang, Y., Liu, Y., Zhu, K., Dong, Y., Cui, H., Mao, L., Zhou, H. (2018). Acute toxicity, antioxidant and antifatigue activities of protein-rich extract from Oviductus ranae. Oxid Med Cell Longev, 2018, 9021371.

Zhang, Y., Wang, Y. F., Li, M. Z., Liu, S. Y., YU, J. L., Yan, Z. W., \& Zhou, H. L. (2019). Traditional uses, bioactive constituents, biological functions and safety properties of Oviductus ranae as functional foods in China. Oxid Med Cell Longev, 2019(21), 1-24. 